\title{
The 27th International Conference on Case-Based Reasoning
}

\author{
Klaus-Dieter Althoff, Kerstin Bach, Ralph Bergmann, Cindy Marling
}

- The 27th International Conference on Case-Based Reasoning was held September 8-12, 2019, in Otzenhausen, Germany. The theme of the conference was explainable artificial intelligence. The conference featured four invited talks, an invited panel, four workshops, a doctoral consortium, four technical paper sessions, and a poster and system demo session. This report summarizes conference highlights.
$\mathrm{F}$ rom September 8 to 12, 2019, approximately 70 research4 ers gathered at the European Academy of Otzenhausen, 1 in Otzenhausen, Germany, for the 27th International Conference on Case-Based Reasoning (ICCBR 2019). The theme of ICCBR 2019 was explainable artificial intelligence (AI). Explanation has been a core aspect of case-based reasoning (CBR) since its very beginning. It has become increasingly important, as the AI community strives to ensure that its systems are understandable, transparent, and trustworthy. The explainable AI theme was explored through four invited talks, a dedicated workshop, and several paper presentations.

The CBR community also marked the return of their premier annual conference to Otzenhausen, Germany, site of the first conference in the ICCBR series in 1993. In 1993, 
the conference was known as the European Workshop on Case-Based Reasoning. In 2002, it was renamed the European Conference on Case-Based Reasoning, and in 2010, it merged with the International Conference on Case-Based Reasoning. The European Academy of Otzenhausen, a remote conference center nestled in the woodlands of Saarland, provided a peaceful backdrop for sharing research, reflecting, brainstorming, and networking.

The conference began with the workshop program and the doctoral consortium. Workshop chairs Hayley Borck and Stelios Kapetanakis assembled a program of four workshops: Case-Based Reasoning for the Explanation of Intelligent Systems, Case-Based Reasoning and Deep Learning, Process-Oriented Case-Based Reasoning, and Case-Based Reasoning in the Health Sciences. Doctoral consortium chairs Antonio SánchezRuiz and Michael Floyd provided a forum for doctoral students to share and obtain feedback on their research and career goals. Each participating student had the opportunity to present their own research, mentored by a senior CBR researcher.

The first invited speaker, David Aha, set the stage for the conference theme of explainable AI and kicked off the Case-Based Reasoning for the Explanation of Intelligent Systems workshop. He presented a broad overview of current explainable AI research and discussed the status of the Defense Advanced Research Projects Agency's explainable AI program. In the next invited talk, Belén Díaz Agudo described the challenges and opportunities for the CBR community in explainable AI. She noted that CBR can exploit memory-based techniques to generate explanations for systems built using different AI techniques, in a broad range of application domains. Andreas Dengel gave the next invited talk, exploring the interpretability and explanatory capacity of deep neural networks. He discussed approaches that attempt to understand and explain how decisions are made by deep neural networks. He noted that it is especially important to be able to explain a system's decisions in domains like autonomous driving or credit allocation, where black box decisions can be risky or unacceptable to people affected by the decisions. The final invited speaker was Daniele Magazzeni. The focus of his talk was model-based reasoning for explainable AI as a service. He discussed this approach in the context of explainable planning, particularly for applications in which human-AI teaming is involved.

The invited panel featured Agnar Aamodt, KlausDieter Althoff, Jean Lieber, Enric Plaza, and Barry Smyth. These senior CBR community members, who attended the first EWCBR-ICCBR conference in Otzenhausen in 1993, were invited to reminisce, reflect upon progress over the past 26 years, and prognosticate advances for the next 26 years. After brief opening statements, questions were taken from the floor and a lively discussion ensued.

The papers selected during the peer review process were presented during four technical paper sessions plus a demo and poster session. The technical paper sessions were organized around the following topics: explainable AI; similarity and analogy; maintenance and adaptation; and community analysis, applications, and learning. There was also a poster and demos pitch session, in which each poster and demo presenter had three minutes to advertise their posters and demos to the community as a whole. The Best Paper Award was presented to Eoin Kenny, Elodie Ruelle, Anne Geoghegan, Laurence Shalloo, Micheál O’Leary, Michael O'Donovan, and Mark Keane for their paper Predicting Grass Growth for Sustainable Dairy Farming: A CBR System Using Bayesian Case-Exclusion and Post-Hoc, Personalized Explanation-by-Example.

A social program rounded out the conference, providing time for continued discussions, networking, and community building. Social events capitalized on the regional offerings surrounding the European Academy of Otzenhausen. Included were a walk and talk through the woods, culminating in a visit to a reconstructed iron-age Celtic village, a barbecue, and a wine tasting, led by local family vintners.

The local and program chairs want to thank the conference sponsors: the Artificial Intelligence Journal, the German Research Center for Artificial Intelligence, IBM, the Knexus Research Corporation, the Norwegian Open Artificial Intelligence Lab, the Norwegian University of Science and Technology, Ohio University, the University of Hildesheim, and the University of Trier. They are also grateful to all of the organizational chairs, as well as to the advisory council and program committee members, who helped to make ICCBR 2019 a success.

The conference proceedings were published by Springer (doi.org/10.1007/978-3-030-29249-2) as part of the Lecture Notes in Computer Science book series (volume 11680). The next ICCBR conference will be held in June 2020, in Salamanca, Spain.

Klaus-Dieter Althoff is a professor in the institute of computer science at the University of Hildesheim, in Hildesheim, Germany. He is also head of the competence center for case-based reasoning at the German Research Center for Artificial Intelligence.

Kerstin Bach is an associate professor in the department of computer science at the Norwegian University of Science and Technology, in Trondheim, Norway. She is the chair of the German Society for Computer Science's Special Interest Group on Knowledge Management and a board member of the Norwegian Artificial Intelligence Society.

Ralph Bergmann is University Professor for business information systems at the University of Trier, in Trier, Germany, where he is head of a research group on artificial intelligence and intelligent information systems. His research focus is on experience-based learning systems, combining casebased reasoning, machine learning, and semantic technologies in various application areas.

Cindy Marling is an associate professor in the school of electrical engineering and computer science at Ohio University, in Athens, Ohio, USA. She is a senior member of Association for the Advancement of Artificial Intelligence. Her research interests include smart and connected health, intelligent decision support, and case-based reasoning. 